\title{
ESTIMATION OF HERITABILITY AND GENETIC ADVANCE TO DEVELOP DROUGHT TOLERANCE IN COTTON (GOSSYPIUM HIRSUTUM L.)
}

\author{
YAR, M. M. ${ }^{1 *}-$ IQBAL M. $^{1}-$ MEHMOOD, A. ${ }^{2}-$ NAEEM, $M .{ }^{1}$ \\ ${ }^{I}$ Depatment of Plant Breeding \& Genetics, University College of Agriculture \& Environmental \\ Sciences, The Islamia University of Bahawalpur, Bahawalpur, Pakistan \\ ${ }^{2}$ Ayub Agricultural Research Institute, Faisalabad, Pakistan \\ *Corresponding author \\ e-mail: majidabbasi1998@gmail.com \\ (Received $3^{\text {rd }}$ Dec 2019; accepted $22^{\text {nd }}$ May 2020)
}

\begin{abstract}
Water scarcity is a major constraint to sustainable cotton production worldwide. Therefore, development of drought tolerant cotton varieties is the main objective of the cotton breeding program. A reduction has been observed in seed cotton yield in cotton plants under drought condition. To evaluate the genetic material under normal and drought conditions, three generations $\left(18 \mathrm{~F}_{2}, 18 \mathrm{BC}_{1}\right.$ and $\left.18 \mathrm{BC}_{2}\right)$, and two parents $\left(6 \mathrm{P}_{1}\right.$ and $\left.3 \mathrm{P}_{2}\right)$ were studied for heritability and genetic advance estimation. $\mathrm{F}_{2}, \mathrm{BC}_{1}$ and $\mathrm{BC}_{2}$ populations were derived through cross combinations of nine parental varieties of upland cotton (Gossypium hirsutum L.). Data were recorded for plant height, number of monopodial branches per plant, number of sympodial branches per plant, number of bolls per plant, boll weight, seed cotton yield per plant, lint percentage and seed index. Heritability estimates ranged from low to high for all traits. Results represent that all studied traits can be improved easily due to high heritability values and phenotypic variations. IUB-09 $\times$ FH-1000 and $\mathrm{BH}-160 \times \mathrm{MNH}-129$ had maximum broad sense, narrow sense heritability and genetic advance for various characters under drought conditions. These two crosses may further increase until later generation to develop drought tolerant cotton cultivars.
\end{abstract}

Keywords: broad sense heritability, narrow sense heritability, water deficit, seed cotton yield

\section{Introduction}

Cotton is the most important cash and fiber crop of global importance (Anderson and Rajasekaran, 2016), cultivated in tropical and subtropical areas of more than 60 different countries (Feng et al., 2017). Cotton crop has $0.8 \%$ share in GDP and gives $4.5 \%$ in value addition of agriculture. Better cotton genotypes are desirable to progress the cotton business although cotton yield, high ginning out turn (GOT) and fine fiber quality are these parameters affect cotton fiber price in the world market (Anonymous 2018-19).

Drought stress reduced the total plant stature up to $35 \%$, decreased leaf area index caused $8 \%$ reduction in solar radiation interception (Pettigrew, 2004). Saranga et al. (1991) observed that the change in water potential explains $87 \%$ change in plant height. The whole phenomenon of drought stress and growth reduction affects total yield of the crop (Pettigrew, 2004; Babar et al., 2009). Boll development is the most critical stage in cotton which is affected by drought stress (Radin et al., 1992). Krieg (2000) mentioned the most critical period for drought stress, in terms of final yield, is from square initiation to first flower. Drought tolerance in cotton is a quantitatively genetically controlled trait (Ahmad et al., 2009). Cotton cultivars display variable genetic response for physiological and morphological parameters under water deficit environments (Hinze et al., 2012; Patil et al., 2017). 
Heritability values provide information about extent of transmission of traits to subsequent generation and response to selection. High heritability represents greater selection response. Heritability estimates will help for effective selection, therefore traits selection on the base of high heritability makes the improvement easier. If environmental influence is small as compare to genetic differences, the selection will be more effective (Khan, 2001). Statistical parameters like mean, variance, genetic advance and heritability support in measuring genetic potential, genetic diversity and stability of any variety (Firouzian, 2003; Ali et al., 2008). Various cotton breeders studied heritability and genetic advance to evaluate hybrid population (Ahsan et al., 2015; Jarwar et al., 2018; Gnanasekaran et al., 2018; Nawaz et al., 2019; Riaz et al., 2019).

The aim of current study is to estimate variability and genetic parameters for yield and other agronomic traits and to investigate new sources of drought tolerance among the segregating populations. These estimates will help in effective selection for improvement of studied traits.

\section{Materials and methods}

The experimental work was conducted during the year 2018, at research area of Department of Plant Breeding \& Genetics, University College Agriculture and Environmental Sciences, The Islamia University of Bahawalpur, Pakistan. Eighty (80) cotton genotypes were evaluated under drought stress at seedling stage by using root and shoot traits. From screening study, six drought tolerant (IUB-09, B-557, 149-F, BH160, BH-118 and FH-900) and three drought susceptible (MNH-129, FH-1000 and FH901) cotton genotypes were selected.

Next year, six drought tolerant (IUB-09, B-557, 149-F, BH-160, BH-118 and FH900) and three drought susceptible (MNH-129, FH-1000 and FH-901) cotton genotypes were crossed in line $\times$ tester (Kempthorne, 1957) scheme to develop 18 cross combinations. Total 27 experimental entries comprising of 9 parents (six lines and three testers) and their $18 \mathrm{~F}_{1}$ hybrids were sown. The experiment was performed in RCBD with 3 replications under normal and drought stress conditions.

\section{Development of $\mathrm{F}_{2}, \mathrm{BC}_{1}$ and $\mathrm{BC}_{2}$ generations}

At maturity $F_{1}$ plants of eighteen crosses were selfed. These selfed seeds were picked individually as a source of $F_{2}$ generation. $F_{1}$ plants were crossed with $1^{\text {st }}$ parent $\left(\mathrm{P}_{1}\right)$ of a specific cross to produce $\mathrm{BC}_{1}$; in the same way they were also crossed with $2^{\text {nd }}$ parent $\left(\mathrm{P}_{2}\right)$ male parent to produce $\mathrm{BC}_{2}$ generations.

\section{Evaluation of $F_{2}, B C_{1}$ and $B C_{2}$ generation}

The seed of nine parents, $18 \mathrm{~F}_{2}, 18 \mathrm{BC}_{1}$ and $18 \mathrm{BC}_{2}$ were planted in field under split plot design. There were two factors i.e. generations and parents and water stress levels. Drought stress was developed after 30 days of sowing. During crop season, drought stress was imposed by supplying 50\% less irrigations till crop maturity in the drought treatment (Kirda et al., 2005). The total irrigation water applied to well water and waterdeficit condition was 24 acre inches and 12 acre inches respectively. The amount of rainfall, maximum and minimum temperature during the crop duration are given in Table 1. The normally irrigated plot was watered when required. For drought stressed plot, irrigation water was applied at critical growth stages of cotton plant i.e. 
germination and emergence, seedling establishment, canopy development, flowering and boll setting. Water stress levels were applied to the main plots while parents and generations were allocated to the sub plots. Each entry was planted in row to row distance $75 \mathrm{~cm}$ whereas plant to plant distances were $30 \mathrm{~cm}$.

Table 1. Monthly average temperature along with total rainfall at experimental site

\begin{tabular}{c|c|c|c}
\hline Month & $\mathbf{T m a x}\left({ }^{\circ} \mathbf{C}\right)$ & $\mathbf{T m i n}\left({ }^{\circ} \mathbf{C}\right)$ & Rainfall $(\mathbf{m m})$ \\
\hline April & 37 & 22.4 & 3.05 \\
May & 39.3 & 26.1 & 8.13 \\
June & 39.8 & 29 & 18.8 \\
July & 38.3 & 28.9 & 56.9 \\
August & 37.2 & 28.4 & 10.93 \\
September & 36.2 & 25.8 & 0 \\
October & 33.6 & 19.6 & 0 \\
November & 27.5 & 14 & 0 \\
\hline
\end{tabular}

Tmax = average maximum temperature; Tmin = average minimum temperature

All the cultural practices were adopted according to routine production technology except drought experiment. Data were recorded on individual plant basis for parents, $\mathrm{BC}_{1}, \mathrm{BC}_{2}$ and $\mathrm{F}_{2}$ generations. For the parents and $\mathrm{F} 1$, data were recorded on 10 randomly selected plants in each replication for each trait. For F2 and backcross generations, data were recorded from 50 and 30 randomly selected plants respectively in each replication.

Data were recorded for Plant height $(\mathrm{cm})$, Number of monopodial branches per plant, Number of sympodial branches per plant, Number of Bolls per Plant, Boll Weight (g), Seed cotton yield per plant (g), Lint Percentage, Seed index.

The obtained lint from each sample was weighed and lint percentage was calculated by using following formula:

$$
\text { Lint percentage }(\%)=\frac{\text { Weight of lint }}{\text { weight of seed cotton }} \times 100
$$

To calculate the seed index, 100 seeds were taken at random for each sample and weighed in gram.

\section{Statistical analysis}

Heritability and genetic advance

Broad sense heritability was computed as described by Mahmud and Kramer (1951).

$$
H^{2}{ }_{\text {(B.S) }}=\frac{V F 2-\sqrt{V P 1} \times V P 2}{V F_{2}} \times 100
$$

where: $\mathrm{H}^{2}{ }_{\mathrm{BS}}=$ broad sense heritability; $\mathrm{VF}_{2}=$ variance of $\mathrm{F}_{2}$ population; $\mathrm{VP}_{1}=$ variance of Parent 1; $\mathrm{VP}_{2}=$ variance of parent 2.

Heritability in narrow sense were computed using the following formula suggested by warner (1952). 


$$
H_{(n, s)}^{2}=\frac{2 V F_{2}-\left(V B C_{1}+V B C_{2}\right)}{V F_{2}} \times 100
$$

where: $\mathrm{H}^{2}{ }_{(\mathrm{n} . \mathrm{s})}=$ heritability in narrow sense; $\mathrm{VF}_{2}=$ variance of $\mathrm{F}_{2}$ population, $\mathrm{VBC}_{1}=$ variance of back cross-I population, $\mathrm{VBC}_{2}=$ variance of back cross-II population.

Genetic advance was calculated by following formula suggested by Allard (1960).

$$
\text { G.A }=\sigma \rho \times \mathrm{h} 2 \times \mathrm{I}
$$

where: $\mathrm{GA}=$ genetic advance; $\sigma \rho=$ phenotypic standard deviation of $\mathrm{F}_{2}$ population; $\mathrm{h} 2$ = estimation of broad sense heritability in fraction, $\mathrm{I}=$ constant value that reflects the selection intensity. The value of $\mathrm{I}=1.755$ in this study the selection pressure is $10 \%$.

\section{Results}

\section{Heritability and genetic advance}

Heritability and genetic advance are remarkable selection parameters. Heritability and genetic advance predict the genetic gain for selection (Johanson et al., 1955). Heritability and genetic advance for different yield traits are as follows.

\section{Plant height}

Under normal condition, broad sense heritability estimates were ranged from $45.77 \%$ $(\mathrm{BH}-118 \times \mathrm{MNH}-129)$ to $93.13 \%$ (IUB-09 $\times \mathrm{FH}-1000)$. Heritability values in narrow sense were ranged from $20.79 \%$ (FH-900 $\times$ FH-1000) to $73.45 \%$ (IUB-09 $\times$ FH-1000). Genetic advance values were ranged from 2.13 (BH-118 $\times$ FH-901) to 11.25 (IUB-09 $\times$ FH-1000). Under drought condition, broad sense heritability values were ranged from $35.59 \%(\mathrm{BH}-118 \times \mathrm{FH}-901)$ to $94.06 \%$ (IUB-09 $\times$ FH-1000). Heritability values in narrow sense were ranged from $17.82 \%(149 \mathrm{~F} \times \mathrm{MNH}-129)$ to $68.51 \%$ (IUB-09 $\times \mathrm{FH}-$ 1000). Genetic advance values were ranged from $1.08(\mathrm{BH}-118 \times \mathrm{FH}-901)$ to 9.24 (IUB-09 × FH-1000) (Table 2).

Table 2. Heritability (broad and narrow sense) and genetic advance for plant height under

\begin{tabular}{|c|c|c|c|c|c|c|}
\hline \multirow{2}{*}{ Crosses } & \multicolumn{3}{|c|}{ Normal condition } & \multicolumn{3}{|c|}{ Drought condition } \\
\hline & $\mathbf{H}^{2}{ }_{\text {bs }}$ & $\mathbf{H}^{2}{ }_{\mathrm{ns}}$ & G.A. & $\mathbf{H}^{2}{ }_{\text {bs }}$ & $\mathbf{H}^{2} \mathrm{~ns}$ & G.A. \\
\hline B-557 $\times$ MNH-129 & 65.58 & 37.05 & 4.02 & 66.75 & 40.34 & 3.05 \\
\hline B-557 × FH-1000 & 64.88 & 38.16 & 3.74 & 58.82 & 39.61 & 2.45 \\
\hline B-557 × FH-901 & 54.23 & 41.40 & 2.57 & 60.44 & 21.04 & 2.46 \\
\hline $149 \mathrm{~F} \times \mathrm{MNH}-129$ & 50.52 & 45.34 & 2.62 & 72.35 & 17.82 & 3.34 \\
\hline $149 \mathrm{~F} \times \mathrm{FH}-1000$ & 53.28 & 48.85 & 2.70 & 63.37 & 48.14 & 2.57 \\
\hline $149 \mathrm{~F} \times \mathrm{FH}-901$ & 69.03 & 23.75 & 4.04 & 72.81 & 54.24 & 3.30 \\
\hline BH-160 $\times$ MNH-129 & 64.57 & 38.97 & 3.42 & 76.53 & 25.35 & 3.50 \\
\hline BH-160 × FH-1000 & 63.76 & 39.69 & 3.17 & 75.68 & 25.75 & 3.44 \\
\hline BH-160 × FH-901 & 92.34 & 61.69 & 9.40 & 93.71 & 62.05 & 8.06 \\
\hline IUB-09 $\times$ MNH-129 & 68.03 & 34.64 & 4.01 & 76.30 & 24.78 & 3.71 \\
\hline
\end{tabular}
normal and drought conditions 


\begin{tabular}{c|c|c|c|c|c|c} 
IUB-09 $\times$ FH-1000 & 93.13 & 73.45 & 11.25 & 94.06 & 68.51 & 9.24 \\
IUB-09 × FH-901 & 59.15 & 39.74 & 2.75 & 65.66 & 36.08 & 2.58 \\
BH-118 × MNH-129 & 45.77 & 45.34 & 2.27 & 68.04 & 25.09 & 3.01 \\
BH-118 × FH-1000 & 51.88 & 33.57 & 2.60 & 49.01 & 29.74 & 1.74 \\
BH-118 × FH-901 & 47.31 & 36.30 & 2.13 & 35.59 & 27.66 & 1.08 \\
FH-900 × MNH-129 & 66.54 & 48.14 & 3.99 & 52.07 & 31.00 & 1.93 \\
FH-900 × FH-1000 & 52.16 & 20.79 & 2.48 & 49.71 & 43.00 & 1.82 \\
FH-900 $\times$ FH-901 & 60.24 & 22.28 & 2.96 & 54.65 & 41.59 & 2.03 \\
\hline
\end{tabular}

Var = variance, $\mathrm{H}^{2}{ }_{\mathrm{bs}}=$ broad sense heritability, $\mathrm{H}^{2}{ }_{\mathrm{ns}}=$ narrow sense heritability, G.A. = genetic advance

\section{Number of monopodial branches per plant}

Broad sense heritability estimates were ranged from $42.09 \%(\mathrm{~B}-557 \times \mathrm{MNH}-129)$ to $77 \%$ (IUB-09 $\times$ FH-1000). Heritability values in narrow sense were ranged from $18.10 \%(\mathrm{FH}-900 \times \mathrm{MNH}-129)$ to $73.80 \%($ IUB-09 $\times$ FH-1000). The genetic advance values were ranged from $0.43(\mathrm{BH}-160 \times \mathrm{FH}-1000)$ to $1.10(\mathrm{FH}-900 \times \mathrm{FH}-1000)$ under normal condition. Under drought condition, broad sense heritability estimates were ranged from $49.58 \%(\mathrm{BH}-160 \times \mathrm{FH}-901)$ to $78.12 \%$ (IUB-09 $\times$ FH-1000). Heritability values in narrow sense were ranged from $13.56 \%(\mathrm{~B}-557 \times \mathrm{FH}-1000)$ to $74.25 \%$ (IUB$09 \times \mathrm{FH}-1000)$. The genetic advance values were ranged from $0.50(\mathrm{FH}-900 \times \mathrm{FH}-$ 1000) to 1.15 (IUB-09 $\times$ FH-1000) (Table 3).

Table 3. Heritability (broad and narrow sense) and genetic advance for monopodial branches per plant under normal and drought conditions

\begin{tabular}{|c|c|c|c|c|c|c|}
\hline \multirow{2}{*}{ Crosses } & \multicolumn{3}{|c|}{ Normal condition } & \multicolumn{3}{|c|}{ Drought condition } \\
\hline & $\mathbf{H}^{2}$ bs & $\mathbf{H}^{2}{ }_{n s}$ & G.A. & $\mathbf{H}^{2}{ }_{b s}$ & $\mathbf{H}^{2} \mathrm{~ns}$ & G.A. \\
\hline B-557 $\times$ MNH-129 & 42.09 & 30.73 & 0.48 & 59.84 & 31.42 & 0.67 \\
\hline B-557 × FH-1000 & 50.55 & 29.17 & 0.54 & 60.98 & 13.56 & 0.73 \\
\hline B-557 × FH-901 & 51.54 & 38.13 & 0.58 & 54.84 & 26.29 & 0.72 \\
\hline $149 \mathrm{~F} \times \mathrm{MNH}-129$ & 58.10 & 37.42 & 0.72 & 60.24 & 35.73 & 0.65 \\
\hline $149 \mathrm{~F} \times \mathrm{FH}-1000$ & 57.03 & 34.37 & 0.60 & 64.29 & 20.04 & 0.77 \\
\hline $149 \mathrm{~F} \times \mathrm{FH}-901$ & 50.28 & 36.25 & 0.52 & 56.54 & 25.11 & 0.72 \\
\hline BH-160 $\times$ MNH-129 & 55.27 & 28.28 & 0.59 & 68.18 & 21.97 & 0.85 \\
\hline BH-160 × FH-1000 & 49.76 & 38.00 & 0.43 & 65.80 & 24.62 & 0.83 \\
\hline BH-160 × FH-901 & 64.76 & 27.14 & 0.70 & 49.58 & 25.39 & 0.61 \\
\hline IUB-09 $\times$ MNH-129 & 66.87 & 64.67 & 0.92 & 73.15 & 66.94 & 0.92 \\
\hline IUB-09 × FH-1000 & 77.00 & 73.80 & 1.10 & 78.12 & 74.25 & 1.15 \\
\hline IUB-09 × FH-901 & 57.00 & 34.02 & 0.63 & 51.17 & 14.23 & 0.59 \\
\hline BH-118 $\times$ MNH-129 & 54.10 & 28.74 & 0.67 & 65.87 & 15.16 & 0.75 \\
\hline BH-118 $\times$ FH-1000 & 57.96 & 31.97 & 0.65 & 61.95 & 28.34 & 0.71 \\
\hline BH-118 × FH-901 & 45.15 & 36.09 & 0.46 & 59.94 & 20.89 & 0.79 \\
\hline FH-900 $\times$ MNH-129 & 48.78 & 18.10 & 0.60 & 67.67 & 27.86 & 0.72 \\
\hline FH-900 × FH-1000 & 66.05 & 27.65 & 0.86 & 53.58 & 15.50 & 0.50 \\
\hline FH-900 × FH-901 & 60.51 & 26.44 & 0.76 & 63.02 & 16.56 & 0.78 \\
\hline
\end{tabular}

$\mathrm{H}^{2}{ }_{\mathrm{bs}}=$ broad sense heritability, $\mathrm{H}^{2}{ }_{\mathrm{ns}}=$ narrow sense heritability, G.A. = genetic advance 


\section{Number of sympodial branches per plant}

Under normal condition, broad sense heritability estimates were ranged from $46.26 \%$ $(149 \mathrm{~F} \times \mathrm{FH}-1000)$ to $90.49 \%$ (IUB-09 $\times \mathrm{FH}-1000)$. Heritability values in narrow sense were ranged from $10.29 \%(\mathrm{~B}-557 \times \mathrm{FH}-1000)$ to $78.36 \%(\mathrm{BH}-118 \times \mathrm{FH}-1000)$. The genetic advance values were ranged from $1.77(149 \mathrm{~F} \times \mathrm{FH}-1000)$ to 8.89 (IUB-09 $\times$ FH-1000). Under drought condition, broad sense heritability estimates were ranged from $49.36 \%(\mathrm{FH}-900 \times \mathrm{FH}-901)$ to $86.81 \%$ (IUB-09 $\times$ FH-1000). Heritability values in narrow sense were ranged from $12.70(\mathrm{BH}-160 \times \mathrm{FH}-1000)$ to $81.52 \%$ (IUB-09 $\times$ $\mathrm{FH}-1000)$. The genetic advance values were ranged from $1.89(\mathrm{FH}-900 \times \mathrm{FH}-901)$ to 6.64 (IUB-09 × FH-1000) (Table 4).

Table 4. Heritability (broad and narrow sense) and genetic advance for number of sympodial branches per plant under normal and drought conditions

\begin{tabular}{|c|c|c|c|c|c|c|}
\hline \multirow{2}{*}{ Crosses } & \multicolumn{3}{|c|}{ Normal condition } & \multicolumn{3}{|c|}{ Drought condition } \\
\hline & $\mathbf{H}^{2}{ }_{b s}$ & $\mathbf{H}^{2} \mathrm{~ns}$ & G.A. & $\mathbf{H}^{2}$ bs & $\mathbf{H}^{2} \mathrm{~ns}$ & G.A. \\
\hline B-557 × MNH-129 & 65.16 & 14.49 & 3.14 & 68.13 & 18.27 & 3.18 \\
\hline B-557 × FH-1000 & 57.31 & 10.29 & 2.56 & 64.27 & 29.79 & 3.32 \\
\hline B-557 × FH-901 & 61.79 & 16.71 & 2.67 & 57.44 & 20.35 & 2.32 \\
\hline $149 \mathrm{~F} \times \mathrm{MNH}-129$ & 54.92 & 13.11 & 2.24 & 64.35 & 13.59 & 3.21 \\
\hline $149 \mathrm{~F} \times \mathrm{FH}-1000$ & 46.26 & 24.24 & 1.77 & 59.97 & 19.37 & 3.31 \\
\hline $149 \mathrm{~F} \times \mathrm{FH}-901$ & 55.24 & 25.27 & 2.12 & 62.63 & 22.61 & 3.05 \\
\hline BH-160 × MNH-129 & 61.07 & 23.61 & 3.25 & 71.86 & 22.51 & 3.25 \\
\hline BH-160 × FH-1000 & 46.44 & 20.78 & 2.16 & 58.18 & 12.70 & 2.53 \\
\hline BH-160 × FH-901 & 57.58 & 15.69 & 2.76 & 62.46 & 26.96 & 2.45 \\
\hline IUB-09 $\times$ MNH-129 & 52.64 & 24.25 & 2.26 & 66.40 & 24.48 & 2.71 \\
\hline IUB-09 × FH-1000 & 90.49 & 67.09 & 8.89 & 86.81 & 81.52 & 6.64 \\
\hline IUB-09 × FH-901 & 49.53 & 18.52 & 1.94 & 69.25 & 22.46 & 2.96 \\
\hline BH-118 $\times$ MNH-129 & 54.36 & 31.63 & 2.37 & 60.70 & 21.20 & 2.50 \\
\hline BH-118 × FH-1000 & 89.58 & 78.36 & 8.35 & 82.92 & 71.08 & 6.08 \\
\hline BH-118 × FH-901 & 65.19 & 24.95 & 3.05 & 62.78 & 15.68 & 2.66 \\
\hline FH-900 $\times$ MNH-129 & 74.04 & 20.88 & 4.82 & 53.47 & 21.66 & 2.14 \\
\hline FH-900 × FH-1000 & 52.48 & 25.98 & 2.59 & 58.93 & 20.31 & 2.95 \\
\hline FH-900 × FH-901 & 48.52 & 24.25 & 2.11 & 49.36 & 19.65 & 1.89 \\
\hline
\end{tabular}

$\mathrm{H}^{2}$ bs $=$ broad sense heritability, $\mathrm{H}^{2}{ }_{\mathrm{ns}}=$ narrow sense heritability, G.A. = genetic advance

\section{Number of bolls per plant}

Under normal condition, broad sense heritability values were ranged from $43.53 \%$ $(\mathrm{BH}-118 \times \mathrm{MNH}-129)$ to $82.68 \%$ (IUB-09 $\times \mathrm{FH}-1000)$. Heritability values in narrow sense were ranged from $10.59 \%(\mathrm{FH}-900 \times \mathrm{FH}-1000)$ to $75.23 \%$ (IUB-09 $\times$ FH-1000). The genetic advance values were ranged from 4.57 (BH-160 $\times$ FH-1000) to 12.76 (IUB$09 \times \mathrm{FH}-1000)$. Under drought condition, broad sense heritability values were ranged from $49.53 \%(\mathrm{FH}-900 \times$ FH-1000) to $81.12 \%$ (IUB-09 $\times$ FH-1000). Heritability values in narrow sense were ranged from $11.96 \%(\mathrm{FH}-900 \times \mathrm{FH}-901)$ to $82.03 \%$ (IUB-09 $\times$ FH-1000). The genetic advance values were ranged from $3.54(\mathrm{FH}-900 \times \mathrm{FH}-1000)$ to 11.23 (IUB-09 × FH-1000) (Table 5). 
Table 5. Heritability (broad and narrow sense) and genetic advance for number of bolls per plant under normal and drought conditions

\begin{tabular}{|c|c|c|c|c|c|c|}
\hline \multirow{2}{*}{ Crosses } & \multicolumn{3}{|c|}{ Normal condition } & \multicolumn{3}{|c|}{ Drought condition } \\
\hline & $\mathbf{H}^{2}{ }_{\mathrm{bs}}$ & $\mathbf{H}^{2}{ }_{\text {ns }}$ & G.A. & $\mathbf{H}^{2}{ }_{b s}$ & $\mathbf{H}^{2}{ }_{\mathrm{ns}}$ & G.A. \\
\hline B-557 $\times$ MNH-129 & 60.69 & 14.11 & 7.54 & 77.54 & 19.58 & 7.56 \\
\hline B-557 × FH-1000 & 66.23 & 17.65 & 8.03 & 64.35 & 12.00 & 5.48 \\
\hline B-557 × FH-901 & 62.69 & 42.93 & 6.60 & 66.28 & 30.79 & 5.66 \\
\hline $149 \mathrm{~F} \times \mathrm{MNH}-129$ & 58.99 & 11.30 & 7.29 & 63.60 & 25.70 & 5.35 \\
\hline $149 \mathrm{~F} \times \mathrm{FH}-1000$ & 46.86 & 13.32 & 4.60 & 51.55 & 24.56 & 4.14 \\
\hline 149F × FH-901 & 53.63 & 48.12 & 5.15 & 50.63 & 15.40 & 3.93 \\
\hline BH-160 × MNH-129 & 72.67 & 63.63 & 9.82 & 73.40 & 60.87 & 6.54 \\
\hline BH-160 × FH-1000 & 50.42 & 15.56 & 4.57 & 65.16 & 20.38 & 5.58 \\
\hline BH-160 × FH-901 & 55.29 & 11.48 & 4.82 & 57.43 & 23.33 & 4.34 \\
\hline IUB-09 $\times$ MNH-129 & 52.07 & 21.28 & 5.34 & 59.56 & 29.61 & 5.12 \\
\hline IUB-09 × FH-1000 & 82.68 & 75.23 & 12.76 & 81.12 & 82.03 & 11.23 \\
\hline IUB-09 × FH-901 & 58.62 & 12.32 & 5.34 & 56.94 & 24.26 & 5.09 \\
\hline BH-118 $\times$ MNH-129 & 43.53 & 29.34 & 4.98 & 55.36 & 19.75 & 4.19 \\
\hline BH-118 $\times$ FH-1000 & 66.97 & 23.66 & 9.06 & 62.18 & 30.28 & 5.62 \\
\hline BH-118 × FH-901 & 55.17 & 41.88 & 5.85 & 50.55 & 12.08 & 3.90 \\
\hline FH-900 $\times$ MNH-129 & 52.43 & 16.20 & 6.64 & 66.44 & 14.79 & 5.30 \\
\hline FH-900 $\times$ FH-1000 & 48.45 & 10.59 & 5.33 & 49.53 & 17.38 & 3.54 \\
\hline FH-900 × FH-901 & 55.63 & 20.58 & 6.02 & 56.02 & 11.96 & 4.19 \\
\hline
\end{tabular}

$\mathrm{H}^{2}$ bs $=$ broad sense heritability, $\mathrm{H}_{\mathrm{ns}}^{2}=$ narrow sense heritability, G.A. = genetic advance

\section{Boll weight}

Under normal condition, the heritability values in broad sense were ranged from $45.59 \%(149 \mathrm{~F} \times \mathrm{FH}-1000)$ to $78.11 \%(\mathrm{BH}-160 \times \mathrm{FH}-901)$. Heritability values in narrow sense were ranged from $9.67 \%(\mathrm{FH}-900 \times \mathrm{FH}-901)$ to $64.04 \%$ (IUB-09 $\times \mathrm{FH}-$ 1000). The genetic advance values were ranged from $0.55(\mathrm{BH}-118 \times \mathrm{MNH}-129)$ to 1.61 (IUB-09 $\times$ FH-1000). Under drought condition, broad sense heritability values were ranged from $28.26 \%(\mathrm{FH}-900 \times \mathrm{FH}-1000)$ to $71.97 \%(\mathrm{~B}-557 \times \mathrm{MNH}-129)$. Under drought condition, heritability values in narrow sense were ranged from $10.12 \%$ (IUB$09 \times \mathrm{FH}-901)$ to $65.75 \%(\mathrm{~B}-557 \times \mathrm{MNH}-129)$. The genetic advance values were ranged from $0.37(\mathrm{FH}-900 \times \mathrm{FH}-1000)$ to $1.50(\mathrm{~B}-557 \times \mathrm{MNH}-129)($ Table 6$)$.

\section{Seed index}

Under normal condition, broad sense heritability values were ranged from $44.06 \%$ $(149 \mathrm{~F} \times \mathrm{FH}-1000)$ to $79.32 \%$ (IUB-09 $\times \mathrm{FH}-1000)$. Heritability values in narrow sense were ranged from $10.24 \%(\mathrm{FH}-900 \times \mathrm{MNH}-129)$ to $72.14 \%$ (IUB-09 $\times \mathrm{FH}-1000)$. The genetic advance values were ranged from $0.96(\mathrm{BH}-118 \times \mathrm{FH}-1000)$ to 2.86 (IUB-09 $\times$ FH-1000). Under drought condition, broad sense heritability values were ranged from $51.90 \%(\mathrm{~B}-557 \times \mathrm{MNH}-129)$ to $81.15 \%$ (IUB-09 $\times$ FH-1000). Heritability values in narrow sense were ranged from $10.15 \%$ (IUB-09 $\times$ FH-901) to $75.30 \%$ (IUB-09 $\times$ FH1000). The genetic advance values were ranged from $0.67(\mathrm{~B}-557 \times \mathrm{FH}-1000)$ to 2.23 (IUB-09 × FH-1000) (Table 7). 
Table 6. Heritability (broad and narrow sense) and genetic advance for boll weight under normal and drought conditions

\begin{tabular}{|c|c|c|c|c|c|c|}
\hline \multirow{2}{*}{ Crosses } & \multicolumn{3}{|c|}{ Normal condition } & \multicolumn{3}{|c|}{ Drought condition } \\
\hline & $\mathbf{H}^{2}{ }_{\mathrm{bs}}$ & $\mathbf{H}^{2}{ }^{2}$ & G.A. & $\mathbf{H}^{2}{ }_{b s}$ & $\mathbf{H}^{2}{ }_{n s}$ & G.A. \\
\hline B-557 × MNH-129 & 76.07 & 61.02 & 1.47 & 71.97 & 65.75 & 1.50 \\
\hline B-557 × FH-1000 & 49.69 & 14.38 & 0.78 & 51.62 & 16.68 & 0.88 \\
\hline B-557 × FH-901 & 53.22 & 25.38 & 0.58 & 50.61 & 16.53 & 0.80 \\
\hline $149 \mathrm{~F} \times \mathrm{MNH}-129$ & 55.35 & 31.13 & 0.97 & 52.73 & 21.39 & 0.80 \\
\hline $149 \mathrm{~F} \times \mathrm{FH}-1000$ & 45.59 & 25.33 & 0.85 & 58.07 & 10.80 & 1.01 \\
\hline $149 \mathrm{~F} \times \mathrm{FH}-901$ & 55.90 & 34.54 & 0.77 & 55.98 & 12.68 & 0.88 \\
\hline BH-160 × MNH-129 & 48.13 & 14.59 & 0.56 & 54.21 & 16.99 & 0.76 \\
\hline BH-160 × FH-1000 & 59.53 & 15.62 & 0.93 & 52.72 & 17.24 & 0.78 \\
\hline BH-160 × FH-901 & 78.11 & 34.02 & 1.10 & 55.07 & 43.11 & 0.78 \\
\hline IUB-09 $\times$ MNH-129 & 49.00 & 41.00 & 0.67 & 52.96 & 20.31 & 0.82 \\
\hline IUB-09 $\times$ FH-1000 & 72.84 & 64.04 & 1.61 & 69.06 & 60.07 & 1.42 \\
\hline IUB-09 × FH-901 & 52.96 & 25.09 & 0.59 & 50.70 & 10.12 & 0.77 \\
\hline BH-118 $\times$ MNH-129 & 46.96 & 14.11 & 0.55 & 51.11 & 10.94 & 0.81 \\
\hline BH-118 $\times$ FH-1000 & 55.21 & 29.11 & 0.83 & 52.23 & 11.75 & 0.90 \\
\hline BH-118 $\times$ FH-901 & 67.22 & 15.88 & 0.78 & 51.41 & 11.85 & 0.82 \\
\hline FH-900 $\times$ MNH-129 & 51.62 & 12.86 & 0.62 & 51.74 & 19.42 & 0.76 \\
\hline FH-900 $\times$ FH-1000 & 48.50 & 11.20 & 0.67 & 28.26 & 11.09 & 0.37 \\
\hline FH-900 × FH-901 & 67.54 & 9.67 & 0.78 & 49.81 & 10.48 & 0.72 \\
\hline
\end{tabular}

$\mathrm{H}^{2}{ }_{\mathrm{bs}}=$ broad sense heritability, $\mathrm{H}^{2}{ }_{n s}=$ narrow sense heritability, G.A. = genetic advance

Table 7. Heritability (broad and narrow sense) and genetic advance for seed index under normal and drought conditions

\begin{tabular}{|c|c|c|c|c|c|c|}
\hline \multirow{2}{*}{ Crosses } & \multicolumn{3}{|c|}{ Normal condition } & \multicolumn{3}{|c|}{ Drought condition } \\
\hline & $\mathbf{H}^{2}{ }_{b s}$ & $\mathbf{H}^{2}{ }_{\mathrm{ns}}$ & G.A. & $\mathbf{H}^{2}{ }_{b s}$ & $\mathbf{H}^{2}{ }_{n s}$ & G.A. \\
\hline B-557 × MNH-129 & 61.73 & 15.16 & 1.46 & 51.90 & 18.79 & 0.69 \\
\hline B-557 × FH-1000 & 52.47 & 13.54 & 1.11 & 53.04 & 11.51 & 0.67 \\
\hline B-557 × FH-901 & 51.36 & 15.85 & 1.15 & 56.64 & 18.79 & 0.68 \\
\hline $149 \mathrm{~F} \times \mathrm{MNH}-129$ & 50.62 & 11.10 & 1.27 & 54.92 & 14.76 & 0.85 \\
\hline $149 \mathrm{~F} \times \mathrm{FH}-1000$ & 44.06 & 12.78 & 1.04 & 62.78 & 12.99 & 0.99 \\
\hline $149 \mathrm{~F} \times \mathrm{FH}-901$ & 49.86 & 14.36 & 1.32 & 57.76 & 16.35 & 0.79 \\
\hline BH-160 $\times$ MNH-129 & 51.77 & 11.38 & 1.29 & 67.76 & 13.50 & 1.16 \\
\hline BH-160 × FH-1000 & 50.07 & 17.68 & 1.22 & 77.39 & 70.81 & 1.47 \\
\hline BH-160 × FH-901 & 49.09 & 14.33 & 1.27 & 75.24 & 10.66 & 1.26 \\
\hline IUB-09 $\times$ MNH-129 & 54.33 & 12.14 & 1.32 & 55.95 & 17.49 & 1.08 \\
\hline IUB-09 × FH-1000 & 79.32 & 72.14 & 2.86 & 81.15 & 75.30 & 2.23 \\
\hline IUB-09 × FH-901 & 66.96 & 60.51 & 2.04 & 62.71 & 10.15 & 1.13 \\
\hline BH-118 $\times$ MNH-129 & 50.97 & 26.37 & 1.11 & 55.41 & 10.23 & 0.77 \\
\hline BH-118 $\times$ FH-1000 & 46.19 & 15.12 & 0.96 & 66.90 & 18.97 & 1.01 \\
\hline BH-118 × FH-901 & 48.52 & 18.31 & 1.10 & 58.21 & 22.61 & 0.72 \\
\hline FH-900 $\times$ MNH-129 & 47.21 & 10.24 & 1.11 & 54.93 & 19.33 & 0.86 \\
\hline FH-900 $\times$ FH-1000 & 50.66 & 19.13 & 1.23 & 59.42 & 21.49 & 0.91 \\
\hline FH-900 × FH-901 & 45.61 & 11.80 & 1.12 & 72.42 & 16.51 & 1.24 \\
\hline
\end{tabular}

$\mathrm{H}^{2}$ bs $=$ broad sense heritability, $\mathrm{H}_{\mathrm{ns}}^{2}=$ narrow sense heritability, G.A. = genetic advance 


\section{Seed cotton yield per plant}

Under normal condition, broad sense heritability values were ranged from 56.54\% $(\mathrm{BH}-160 \times \mathrm{MNH}-129)$ to $76.07 \%$ (IUB-09 $\times \mathrm{FH}-1000)$. Heritability values in narrow sense were ranged from $11.10 \%(\mathrm{~B}-557 \times \mathrm{FH}-1000)$ to $62.21 \%$ (IUB-09 $\times$ FH-1000). Genetic advance values were ranged from $10.38(\mathrm{BH}-160 \times \mathrm{MNH}-129)$ to 18.79 (IUB$09 \times \mathrm{FH}-1000)$. Under drought stress condition, broad sense heritability values were ranged from $54.70 \%(\mathrm{~B}-557 \times \mathrm{MNH}-129)$ to $80.33 \%$ (IUB-09 $\times$ FH-1000). Heritability values in narrow sense were ranged from $11.34 \%(\mathrm{BH}-118 \times \mathrm{MNH}-129)$ to $77.24 \%$ (IUB-09 $\times$ FH-1000). Genetic advance values were ranged from $8.73(\mathrm{BH}-118 \times \mathrm{FH}-$ 901) to 19.34 (IUB-09 $\times$ FH-1000) (Table 8).

Table 8. Heritability (broad and narrow sense) and genetic advance for seed cotton yield per plant under normal and drought conditions

\begin{tabular}{|c|c|c|c|c|c|c|}
\hline \multirow{2}{*}{ Crosses } & \multicolumn{3}{|c|}{ Normal condition } & \multicolumn{3}{|c|}{ Drought condition } \\
\hline & $\mathbf{H}^{2}{ }_{\text {bs }}$ & $\mathbf{H}^{2}{ }_{\mathrm{ns}}$ & G.A. & $\mathbf{H}^{2}{ }_{\mathrm{bs}}$ & $\mathbf{H}^{2}{ }_{\mathrm{ns}}$ & G.A. \\
\hline B-557 $\times$ MNH-129 & 60.02 & 21.43 & 11.07 & 54.70 & 22.20 & 9.03 \\
\hline B-557 × FH-1000 & 56.91 & 11.10 & 10.41 & 61.80 & 18.47 & 10.74 \\
\hline B-557 × FH-901 & 61.75 & 13.61 & 11.57 & 70.59 & 24.22 & 13.88 \\
\hline $149 \mathrm{~F} \times \mathrm{MNH}-129$ & 58.72 & 16.52 & 11.56 & 65.38 & 26.10 & 12.23 \\
\hline $149 \mathrm{~F} \times \mathrm{FH}-1000$ & 60.95 & 16.82 & 12.70 & 69.73 & 20.49 & 13.49 \\
\hline $149 \mathrm{~F} \times \mathrm{FH}-901$ & 64.07 & 17.88 & 13.43 & 72.87 & 23.52 & 14.78 \\
\hline BH-160 $\times$ MNH-129 & 56.54 & 18.20 & 10.38 & 75.69 & 15.64 & 15.63 \\
\hline BH-160 × FH-1000 & 68.11 & 61.36 & 15.02 & 77.97 & 70.19 & 16.35 \\
\hline BH-160 × FH-901 & 67.59 & 18.23 & 14.27 & 64.81 & 16.43 & 10.68 \\
\hline IUB-09 $\times$ MNH-129 & 65.90 & 18.67 & 13.25 & 64.89 & 14.80 & 12.10 \\
\hline IUB-09 × FH-1000 & 76.07 & 62.21 & 18.79 & 80.33 & 77.24 & 19.34 \\
\hline IUB-09 $\times$ FH-901 & 68.30 & 58.91 & 14.14 & 73.82 & 69.61 & 15.29 \\
\hline BH-118 $\times$ MNH-129 & 57.57 & 18.20 & 11.42 & 66.35 & 11.34 & 12.26 \\
\hline BH-118 $\times$ FH-1000 & 56.65 & 11.54 & 11.45 & 62.05 & 26.19 & 10.44 \\
\hline BH-118 × FH-901 & 59.32 & 28.61 & 11.94 & 56.16 & 19.71 & 8.73 \\
\hline FH-900 $\times$ MNH-129 & 66.49 & 17.58 & 11.67 & 63.09 & 13.68 & 12.05 \\
\hline FH-900 × FH-1000 & 68.12 & 13.28 & 12.62 & 61.49 & 13.27 & 11.11 \\
\hline FH-900 × FH-901 & 64.23 & 15.18 & 10.84 & 57.73 & 19.73 & 9.89 \\
\hline
\end{tabular}

$\mathrm{H}^{2}{ }_{\mathrm{bs}}=$ broad sense heritability, $\mathrm{H}^{2}{ }_{\mathrm{ns}}=$ narrow sense heritability, G.A. = genetic advance

\section{Lint percentage}

Under normal condition, broad sense heritability values were ranged from $35.91 \%$ $(\mathrm{BH}-160 \times \mathrm{FH}-901)$ to $72.85 \%(\mathrm{BH}-160 \times \mathrm{MNH}-129)$. Heritability values in narrow sense were ranged from $10.06 \%$ (BH-160 $\times$ FH-1000) to $66.50 \%$ (IUB-09 $\times$ FH-1000). Genetic advance values were ranged from $0.84(\mathrm{BH}-160 \times \mathrm{FH}-901)$ to 2.90 (IUB-09 $\times$ FH-1000). Under drought condition, broad sense heritability values were ranged from $47.71 \%$ (IUB-09 $\times$ MNH-129) to $86.54 \%(\mathrm{HB}-160 \times \mathrm{MNH}-129)$. Heritability values in narrow sense were ranged from $10.49 \%(\mathrm{BH}-118 \times \mathrm{MNH}-129)$ to $82.07 \%(\mathrm{BH}-160 \times$ $\mathrm{MNH}-129)$. The genetic advance was ranged from $1.11(\mathrm{BH}-160 \times \mathrm{FH}-1000)$ to 3.55 (IUB-09 $\times$ FH-1000) (Table 9). 
Table 9. Heritability (broad and narrow sense) and genetic advance for lint percentage under normal and drought conditions

\begin{tabular}{|c|c|c|c|c|c|c|}
\hline \multirow{2}{*}{ Crosses } & \multicolumn{3}{|c|}{ Normal condition } & \multicolumn{3}{|c|}{ Drought condition } \\
\hline & $\mathbf{H}^{2}{ }_{\text {bs }}$ & $\mathbf{H}^{2}{ }_{\mathrm{ns}}$ & G.A. & $\mathbf{H}^{2}{ }_{b s}$ & $\mathbf{H}^{2}{ }{ }^{2}$ & G.A. \\
\hline B-557 × MNH-129 & 59.19 & 11.54 & 1.81 & 66.38 & 11.85 & 2.01 \\
\hline B-557 × FH-1000 & 49.54 & 14.58 & 1.65 & 58.95 & 16.31 & 1.58 \\
\hline B-557 × FH-901 & 52.74 & 30.10 & 1.58 & 48.70 & 12.00 & 1.32 \\
\hline $149 \mathrm{~F} \times \mathrm{MNH}-129$ & 53.07 & 17.43 & 1.51 & 58.85 & 13.10 & 1.89 \\
\hline $149 \mathrm{~F} \times \mathrm{FH}-1000$ & 60.43 & 11.49 & 2.26 & 64.92 & 17.57 & 2.20 \\
\hline $149 \mathrm{~F} \times \mathrm{FH}-901$ & 54.41 & 16.43 & 1.66 & 61.17 & 11.35 & 2.24 \\
\hline BH-160 $\times$ MNH-129 & 72.85 & 64.87 & 2.47 & 86.54 & 82.07 & 3.33 \\
\hline BH-160 $\times$ FH-1000 & 47.18 & 10.06 & 1.38 & 54.41 & 34.00 & 1.11 \\
\hline BH-160 × FH-901 & 35.91 & 15.19 & 0.84 & 64.65 & 13.52 & 1.70 \\
\hline IUB-09 $\times$ MNH-129 & 63.18 & 14.30 & 1.84 & 47.71 & 13.14 & 1.29 \\
\hline IUB-09 × FH-1000 & 71.93 & 66.50 & 2.90 & 80.89 & 69.04 & 3.55 \\
\hline IUB-09 × FH-901 & 58.64 & 14.41 & 1.70 & 63.60 & 11.29 & 2.29 \\
\hline BH-118 $\times$ MNH-129 & 59.50 & 14.22 & 1.95 & 67.47 & 10.49 & 1.81 \\
\hline BH-118 $\times$ FH-1000 & 54.36 & 10.87 & 2.03 & 69.68 & 15.03 & 1.89 \\
\hline BH-118 × FH-901 & 57.38 & 13.89 & 1.94 & 71.85 & 62.93 & 2.29 \\
\hline FH-900 $\times$ MNH-129 & 53.23 & 11.28 & 1.53 & 62.09 & 14.16 & 1.65 \\
\hline FH-900 $\times$ FH-1000 & 61.49 & 10.96 & 2.36 & 64.92 & 18.67 & 1.75 \\
\hline FH-900 × FH-901 & 62.76 & 14.91 & 2.14 & 57.03 & 28.35 & 1.57 \\
\hline
\end{tabular}

$\mathrm{H}^{2}$ bs $=$ broad sense heritability, $\mathrm{H}_{\mathrm{ns}}^{2}=$ narrow sense heritability, G.A. = genetic advance

\section{Discussion}

Drought is the most significant abiotic stress manipulating the performance of crop plants. Therefore, screening for identification or development of tolerant varieties is of high importance for enhancing cotton production (Dahab et al., 2012). In order to achieve such evidence in $G$. hirsutum, 18 hybrids were assessed for growth and productivity characters under normal and drought conditions.

Genetic variation is valuable for permanent genetic improvement (Singh, 2000). Most of the crosses of plant height revealed high magnitude of broad sense heritability under normal and drought conditions. Generally moderate to high values of broad and narrow sense heritability exhibited that genetic effects were greater than influence of the environment and characters can be improved by selection (Bnejdi and Gazzah, 2008) while selection on the base of low heritability may be misleading due to more effect of environment on the genetic makeup (Nadarajan and Gunasekaran, 2005). Estimated narrow sense heritability and genetic advance differed for various hybrids under both conditions. Various crosses of plant height exhibited low to moderate narrow sense heritability in both conditions. Crosses with moderate heritability values are indicative of smaller environmental effects which recommends that improvement of the said traits can be easily improved through simple selection procedures (Noor et al., 2017). Batool et al. (2013) and Khan and Hassan (2011) also reported high and moderate heritability for plant height. Under both conditions, IUB-09 $\times$ FH-1000 and BH-160 × FH-901 exhibited maximum high broad sense heritability as well as high narrow sense 
heritability and higher genetic advance for plant height showed that these crosses are controlled by additive genes and selection for these crosses would be valuable.

Most of the crosses of monopodial branches per plant revealed high magnitude of the broad sense heritability under normal and drought conditions. Estimated narrow sense heritability and genetic advance varied for various hybrids in both conditions. Ahmed et al. (2006) and Khan and Hassan (2011) reported high and moderate heritability for number of monopodial branches per plant. Under both conditions, IUB-09 $\times$ FH-1000 and IUB-09 $\times$ MNH-129 exhibited maximum high broad sense heritability as well as high narrow sense heritability and appreciable genetic advance for monopodial branches per plant, suggested that these crosses are controlled by additive genes and thus could lead to development of new genotype by the use of efficient selection techniques.

Most of the crosses of sympodial branches per plant exhibited moderate to higher broad sense heritability estimates. Various crosses exhibited low to moderate narrow sense heritability in both conditions. Low narrow sense heritability may be caused by low additive effects and high dominance gene action (Falconer and mackay, 1996). Soomro et al. (2010) and Khan and Hassan (2011) reported high and moderate heritability values for sympodial branches per plant. Under both conditions, IUB-09 $\times$ FH-1000 and BH-118 $\times$ FH-1000 exhibited maximum high broad and narrow sense heritability and appreciable genetic advance for sympodial branches per plant showed that this cross is controlled by additive genes and selection for these crosses would be effective.

Most of the crosses for bolls per plant revealed high broad sense heritability under normal and drought conditions. Genetic advance and narrow sense heritability estimates varied for different crosses under both conditions. High heritability values with high genetic advance are highly effective in predicting gains under selection in the development of genotypes (Khan et al., 2015). Dhivya et al. (2014) and Ahsan et al. (2015) stated high and moderate heritability values for bolls per plant. Under both conditions, IUB-09 $\times$ FH-1000 and BH-160 $\times$ MNH-129 exhibited maximum high broad sense heritability as well as high narrow sense heritability and appreciable genetic advance for number of bolls per plant showed that these crosses are controlled by additive gene action and selection for these crosses would be valuable.

The low narrow sense heritability might be due to large epistatic effects (Hakizimana et al., 2004). The value of heritability alone does not provide any indication the amount of genetic progress that may lead to selection of best individual, but heritability with genetic advance are more useful (Mishra et al., 2015; Joshi and Patil, 2018). Genetic advance is also of great significance as it shows magnitude of the expected genetic gain from single cycle of selection (Hamdi et al., 2003). Most crosses of boll weight revealed high magnitude of the broad sense heritability under both conditions. Estimated genetic advance and narrow sense heritability differed for different hybrids under both conditions. Jarwar et al. (2018), Gnanasekaran et al. (2018) and Riaz et al. (2019) reported high and moderate heritability values for boll weight. Under both conditions, IUB-09 $\times$ FH-1000 and B-557 $\times$ MNH-129 exhibited maximum high broad sense heritability with high narrow sense heritability and higher genetic advance for boll weight showed that these crosses are controlled by additive gene action and selection for these crosses would be valuable.

Most of the crosses of seed index revealed high magnitude of broad sense heritability under normal and drought conditions. Estimated genetic advance and narrow sense heritability differed for different hybrids under both conditions. Shakeel et al. (2015), 
Shao et al. (2016) and Jarwar et al. (2018) stated high and moderate heritability estimates for seed index. Under normal condition, IUB-09 $\times$ FH-1000 and IUB-09 $\times$ FH-901 whereas under drought condition, IUB-09 $\times$ FH-1000 and BH-160 $\times$ FH-1000 exhibited maximum high broad sense heritability with high narrow sense heritability and higher genetic advance for seed index showed that these crosses are controlled by additive gene action and selection for these crosses would be valuable.

Seed cotton yield per plant is determined from two basic traits i.e. boll number and boll weight. When drought stress occurs during the flowering stage, seed cotton yield decreases due to shedding of squares and young bolls (Cook and El-Zik, 1992). Higher estimates of heritability are not only useful in predicting gain under selection but also indicative of additive gene effects (Farooq et al., 2018). Various crosses of seed cotton yield per plant revealed high magnitude of broad sense heritability under both conditions. Genetic advance and narrow sense heritability differed for different hybrids under both conditions. Riaz et al. (2019) and Nawaz et al. (2019) reported high and moderate heritability estimates for seed cotton yield per plant. Under both conditions, IUB-09 $\times$ FH-1000, BH-160 $\times$ FH-1000 and IUB-09 $\times$ FH-901 exhibited maximum high broad sense heritability with high narrow sense heritability and higher genetic advance for seed cotton yield showed that these crosses are controlled by additive gene action and selection for these crosses would be valuable.

Most of the crosses of lint percentage revealed high magnitude of the broad sense heritability under both conditions. Estimated genetic advance and narrow sense heritability differed for each hybrid under both conditions. Most of the crosses of lint percentage exhibited low to moderate narrow sense heritability in both conditions. Dhamayanthi et al. (2018) and Nawaz et al. (2019) reported high and moderate heritability estimates for lint percentage. Under normal condition, IUB-09 $\times$ FH-1000 and $\mathrm{BH}-160 \times \mathrm{MNH}-129$ whereas under drought condition, IUB-09 $\times \mathrm{FH}-1000, \mathrm{BH}-$ $118 \times$ FH-901 and BH-160 $\times$ MNH-129 exhibited maximum high broad sense heritability with high narrow sense heritability and higher genetic advance for lint percentage showed that these crosses are controlled by additive gene action and selection for these crosses would be valuable.

\section{Conclusions}

From heritability estimates, it is manifest that crosses i.e. IUB-09 $\times$ FH-1000 and BH-160 $\times$ MNH-129 exhibited high heritability $\left(\mathrm{H}^{2}\right.$ b.s $)$ with high genetic advance for plant height, monopodial branches per plant, sympodial branches per plant, bolls per plant, boll weight, seed cotton yield per plant, lint percentage and seed index. It is therefore recommended that varieties involved in these promising hybrids should be given due consideration in further breeding program and selection for these traits could be practiced with due care to achieve desirable level of yield potential in cotton and the highest values of saving water at the same time. Furthermore, the progenies of these crosses may be raised till later generation to develop cotton drought tolerance cultivars.

\section{REFERENCES}

[1] Ahmad, R. T., Malik, T. A., Khan, I. A., Jaskani, M. J. (2009): Genetic analysis of some morpho-physiological traits related to drought strss in cotton (Gossypium hirsutum). International Journal of Agriculture and Biology 1: 235-240. 
[2] Ahmed, H. M., Kandhro, M. M., Laghari, S., Abro, S. (2006): Heritability and genetic advance as selection indicators for improvement in cotton (Gossypium hirsutum L.). Journal of Biological Sciences 6: 96-99.

[3] Ahsan, M. Z., Majidano, M. S., Bhutto, H., Soomro, A. W., Panhwar, F. H., Channa, A. R., Sial, K. B. (2015): Genetic variability, coefficient of variance, heritability and genetic advance of some Gossypium hirsutum L. accessions. - Journal of Agricultural Science 7(2): 147-151.

[4] Ali, Y., Atta, B. M., Akhtar, J., Monneveux, P., Lateef, Z. (2008): Genetic variability, association and diversity studies in wheat (Triticum aestivum L.) germplasm. - Pakistan Journal of Botany 40(5): 2087-2097.

[5] Allard, R. W. (1960): Principles of Plant Breeding. - John Wiley and Sons, Inc., New York.

[6] Anderson, D. M., Rajasekaran, K. (2016): The Global Importance of Transgenic Cotton. - In: Ramawat, K. G., Ahuja, M. R. (eds.) Fiber Plants: Biology, Biotechnology and Applications. Springer International Publishing, Cham, pp. 17-33.

[7] Anonymous (2018-19): Agricultural Statistics of Pakistan. - Government of Pakistan, Ministry of Food, Agriculture and Live Stock, Economic Wing, Islamabad.

[8] Babar, M., Saranga, Y., Iqbal, Z., Arif, M., Zafar, Y., Lubbers, E., Chee, P. (2009): Identification of QTLs and impact of selection from various environments (dry vs irrigated) on the genetic relationships among the selected cotton lines from F6 population using a phylogenetic approach. - African Journal of Biotechnology 8: 4802-4810.

[9] Batool, S., Khan, N. U., Gull, S., Baloch, M. J., Turi, N. A., Taran, S. A., Saeed, M. (2013): Genetic analysis for yield and yield contributing variables in upland cotton. Journal of Food, Agriculture and Environment 11: 624-630.

[10] Bnejdi, F., Gazzah, M. E. 1. (2008): Inheritance of resistance to yellowberry in durum wheat. - Euphytica 163: 225-230.

[11] Cook, C. G., El-Zik, K. M. (1992): Cotton seedling and first-bloom plant characteristics: relationships with drought-influenced boll abscission and lint yield. - Crop Science 32: 1464-1467.

[12] Dahab, A. H. A., Mohamed, B. B., Husnain, T., Saeed, M. (2012): Variability for drought tolerance in cotton (Gossypium hirsutum L.) for growth and productivity traits using selection index. - African Journal of Agricultural Research 7(35): 4934-4942.

[13] Dhamayanthi, K. P. M., Manivannan, A., Saravanan, M. (2018): Evaluation of new germplasm of Egyptian cotton (G. barbadense) through multivariate genetic component analysis. - Electronic Journal of Plant Breeding 9(4): 1348-1354.

[14] Dhivya, R., Amalabalu, P., Pushpa, R., Kavithamani, D. (2014): Variability, heritability and genetic advance in upland cotton (Gossypium hirsutum L.). - African Journal of Plant Science 8(1): 1-5.

[15] Falconer, D. S., Mackay, T. F. C. (1996): Introduction to Quantitative Genetics. 4th Ed. Longman Group Limited, England.

[16] Farooq, J., Rizwan, M., Saleem, S., Sharif, I., Chohan, S. M., Riaz, M., Ilhai, F., Kainth, R. A. (2018): Determination of genetic variation for earliness, yield and fiber traits in advance lines of cotton (Gossypium hirsutum). - Advances in Agricultural Science 6(2): 59-74.

[17] Feng, L., Dai, J., Tian, L., Zhang, H., Li, W., Dong, H. (2017): Review of the technology for high yielding and efficient cotton cultivation in the northwest inland cotton-growing region of China. - Field Crops Research 208: 18-26.

[18] Firouzian, A. (2003): Heritability and genetic advance of grain yield and its related traits in wheat. - Pakistan Journal of Biological Sciences 4(24): 2020-2023.

[19] Gnanasekaran, M., Thiyagu, K., Gunasekaran, M. (2018): Genetic variability heritability and genetic advance studies in cotton (Gossypium hirsutum L.). - Electronic Journal of Plant Breeding 9(1): 377-382. 
[20] Hakizimana, F., Ibrahim, M. H., Langham, A. C., Rudd, C. J., Haley, D. S. (2004): Generation mean analysis of wheat streak mosaic virus resistance in winter wheat. Euphytica. 139: 133-139.

[21] Hamdi, A., El-Ghareib, A. A., Shafey, S. A., Ibrahim, M. A. M. (2003): Genetic variability, heritability and expected genetic advance for earliness and seed yield from selection in lentil. - Egyptian Journal of Agricultural Research 81: 125-137.

[22] Hinze, L. L., Dever. J. K., Percy, R. G. (2012): Molecular Variation among and within improved cultivars in the U.S. cotton germplasm collection. - Crop Science 52: 222-230.

[23] Jarwar, A. H., Wang, X., Wang, L., Ma, Q., Fan, S. (2018): Genetic advancement, variability and heritability in upland cotton (Gossypium hirsutum L.). - Journal of Environmental and Agricultural Sciences 16: 24-31.

[24] Johanson, H. W., Robinson, H. F., Comstock, R. E. (1955): Estimation of genetic and environmental variability in soybean. - Agronomy Journal 47: 314-318.

[25] Joshi, V., Patil, B. R. (2018): Genetic variability and heritability study in F2 population for yield, yield attributes and fibre quality traits in cotton (Gossypium hirsutum L.). Journal of Pharmacognosy and Phytochemistry 7(4): 2816-2818.

[26] Kempthorne, O. (1957): An Introduction to Genetic Statistics. - John Wiley and Sons, Inc., New York.

[27] Khan, M. A. (2001): Experimental design and analysis heritability estimation. - 2: 210211.

[28] Khan, N., Ullah, F., Khalil, I. H., Naheed, H., Abid, S. (2015): Heritability and genetic advance studies for biochemical traits in F2-3 introgressed families of brassica. Pakistan Journal of Botany 47(3): 883-888.

[29] Khan, N. U., Hassan, G. (2011): Genetic effects on morphological and yield traits in cotton (Gossypium hirsutum L.). - Spanish Journal of Agricultural Research 9(2): 460472.

[30] Kirda, C., Topeu, S., Kaman, H., Ulger, A. C., Yazici, A., Cetin, M., Derici, M. R. (2005): Grain yield response and nitrogen fertilizer recovery of maize under deficit irrigation. - Photosynthetica 19: 312-319.

[31] Krieg, D. R. (2000): Cotton Water Relations. Special Report - In: Oosterhuis, D. M. (ed.) Proc. Cotton Research Meeting and Summaries of Cotton Research. Arkansas Agric. Exp. Sta., Fayetteville, AR.

[32] Mahmud, I., Kramer, H. H. (1951): Segregation for yield, height and maturity following a soybean cross. - Agronomy Journal 43: 605-609.

[33] Mishra, P. K., Ram, R. B., Kumar, N. (2015): Genetic variability, heritability, and genetic advance in strawberry (Fragaria $\times$ ananassa Duch.). - Turkish Journal of Agriculture and Forestry 39: 451-458.

[34] Nadarajan, N., Gunasekaran, M. (2005): Quantitative Genetics and Biometrical Techniques in Plant Breeding. - Kalyani Publ., New Delhi.

[35] Nawaz, B., Sattar, S., Malik, T. A. (2019): Genetic analysis of yield components and fiber quality parameters in upland cotton. - International Multidisciplinary Research Journal 9: 13-19.

[36] Noor, M., Rahman, H. U., Iqbal, M. (2017): Heritability estimates for maturity and plant characteristics in popcorn. - Sarhad Journal of Agriculture 33(2): 276-281.

[37] Patil, N. P., Salve, A. N., Adsare, A. D. (2017): Combining ability studies over environments for sucking pest and yield in upland cotton. - Journal of Global Biosciences 6(4): 4918-4934.

[38] Pettigrew, W. T. (2004): Physiological consequences of moisture deficit stress in cotton. - Crop Science 44: 1265-1272.

[39] Radin, J. W., Mauney, J. R., Reaves, L. L., French, O. F. (1992): Yield enhancement by frequent irrigation during fruiting. - Agronomy Journal 84: 551-557. 
[40] Riaz, B., Saeed, A., Fiaz, S., Riaz, A. (2019): Genetic diversity among Bt cotton (Gossypium hirsutum L.) germplasm assessed through morphological and within boll yield attributes. - Journal of Animal and Plant Sciences 29(1): 226-231.

[41] Saranga, Y., Rudich, J., Marani, A. (1991): The relations between leaf water potential of cotton plants and environmental and plant factors. - Field Crops Research 28: 39-46.

[42] Shakeel, A., Talib, I., Rashid, M., Saeed, A., Ziaf, K., Saleem, M. F. (2015): Genetic diversity among upland cotton genotypes for quality and yield related traits. - Pakistan Journal of Agricultural Sciences 52(1): 73-77.

[43] Shao, D., Wang, T., Zhang, H., Zhu, J., Tang, F. (2016): Variation, heritability and association of yield, fiber and morphological traits in a near long staple upland cotton population. - Pakistan Journal of Botany 48(5): 1945-1949.

[44] Singh, B. D. (2000): Plant Breeding: Principles and Methods. - Kalyani Publishers, New Delhi.

[45] Soomro, Z. A., Kumbhar, M. B., Larik, A. S., Imran, M., Brohi, S. A. (2010): Heritability and selection response in segregating generations of upland cotton. - Pakistan Journal of Agricultural Research 23(1): 25-30.

[46] Warner, J. N. (1952): A method for estimating heritability. - Agronomy Journal 44: 427430. 